Revue des patrimoines

13 | 2010

Art rupestre : la 3D un outil de médiation du réel invisible?

\title{
De la frise magdalénienne in situ ... au centre d'interprétation du Roc-aux-Sorciers : l'usage de la 3D
}

Geneviève Pinçon, Oscar Fuentes, René Barré, Olivier Auber et Gaël Hamon

\section{OpenEdition}

\section{Journals}

Édition électronique

URL : http://journals.openedition.org/insitu/6672

DOI : 10.4000/insitu.6672

ISSN : $1630-7305$

Éditeur

Ministère de la Culture

Référence électronique

Geneviève Pinçon, Oscar Fuentes, René Barré, Olivier Auber et Gaël Hamon, « De la frise

magdalénienne in situ ... au centre d'interprétation du Roc-aux-Sorciers : I'usage de la 3D », In Situ [En

ligne], 13 | 2010, mis en ligne le 16 avril 2012, consulté le 14 novembre 2019. URL : http:// journals.openedition.org/insitu/6672; DOI : 10.4000/insitu.6672

Ce document a été généré automatiquement le 14 novembre 2019.

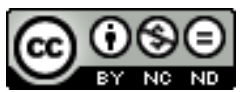

In Situ Revues des patrimoines est mis à disposition selon les termes de la licence Creative Commons Attribution - Pas d'Utilisation Commerciale - Pas de Modification 4.0 International. 


\title{
De la frise magdalénienne in situ ... au centre d'interprétation du Roc- aux-Sorciers : l'usage de la 3D
}

\author{
Geneviève Pinçon, Oscar Fuentes, René Barré, Olivier Auber et Gaël Hamon
}

\section{Le Roc-aux-Sorciers : un site unique au monde à étudier et faire connaître}

1 Au point de contact du Poitou, de la Touraine et du Berry, dans le département de la Vienne, la commune d'Angles-sur-l'Anglin abrite l'un des joyaux de l'art pariétal paléolithique européen : la frise sculptée magdalénienne du Roc-aux-Sorciers. Le site du Roc-aux-Sorciers se situe à $1,5 \mathrm{~km}$ du bourg d'Angles-sur-l'Anglin. De par sa richesse thématique, stylistique et technique, mais aussi par son ampleur et son état de conservation exceptionnel, ce site majeur de la préhistoire européenne, classé au titre des monuments historiques le 18 janvier 1955, est souvent cité comme le «Lascaux de la sculpture $»^{1}$.

2 Après la découverte de l'occupation magdalénienne au lieu-dit «Le Roc-aux-Sorciers » par Lucien Rousseau en $1927^{2}$, nous devons à Suzanne Cassou de Saint-Mathurin et Dorothy Garrod la mise au jour dans cet abri sous roche des œuvres sculptées, gravées et peintes, lors des fouilles qu'elles ont réalisées entre 1947 et 1964. Dégagées dans leur contexte archéologique, ces œuvres sont particulièrement bien datées, il y a 15000 ans et attribuables au Magdalénien moyen, une culture de la fin du Paléolithique ${ }^{3} 567$. 
Figure 1

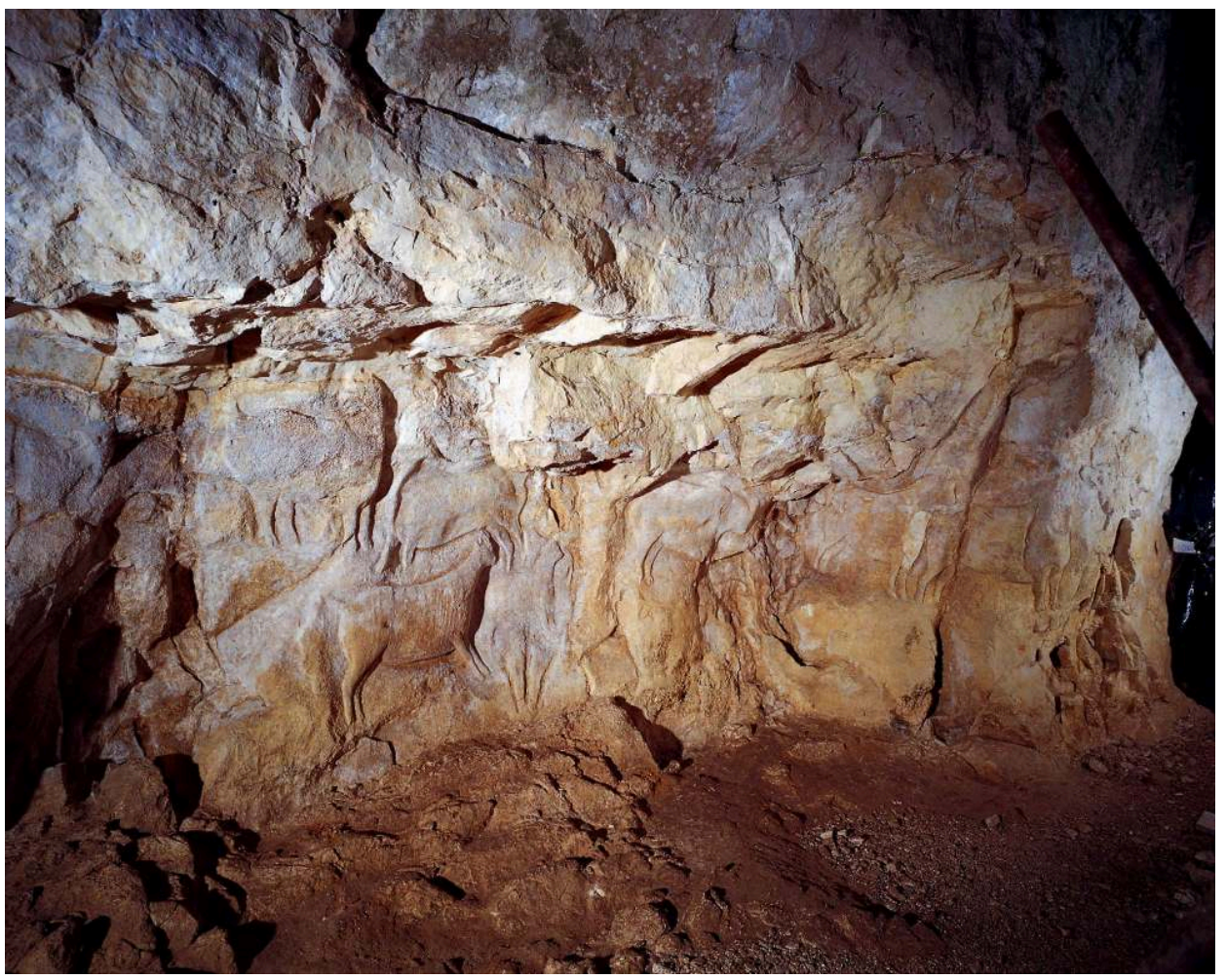

Panneau des bouquetins sculptés grandeur nature

(c) Geneviève Pinçon, ministère de la Culture et de la Communication, phot. Alain Maulny

3 Dans la partie amont du site, dite la cave Taillebourg, elles mettent progressivement au jour, dès 1947, des blocs ornés provenant du plafond effondré, puis un bison sculpté encore in situ dans ce secteur. Plus en aval, dans l'abri Bourdois, elles découvrent en 1950 un cheval sculpté, encore en place sur la paroi, en fond d'abri et découvrent alors sur près de 20 mètres de long, in situ, des gravures discrètes mais aussi des sculptures monumentales de bouquetins, bisons, chevaux, félins et plusieurs représentations humaines, dont trois femmes. Celles-ci, sculptées grandeur nature comme les bouquetins, n'ont pas d'équivalent dans le monde de l'art paléolithique (fig. $\mathbf{n}^{\circ} \mathbf{1}$ ).

4 À son décès survenu le 25 août 1991, Suzanne Cassou de Saint-Mathurin lègue à l'État, par voie testamentaire, le site et ses archives et collections (Musée d'archéologie national), sans en avoir terminé l'étude, la publication et la restitution au public comme elle le souhaitait. Il nous est alors revenu de poursuivre ce travail et c'est tout d'abord dans ce cadre d'analyse scientifique que l'utilisation de la 3D a été envisagée ${ }^{8}$. 
Figure 2

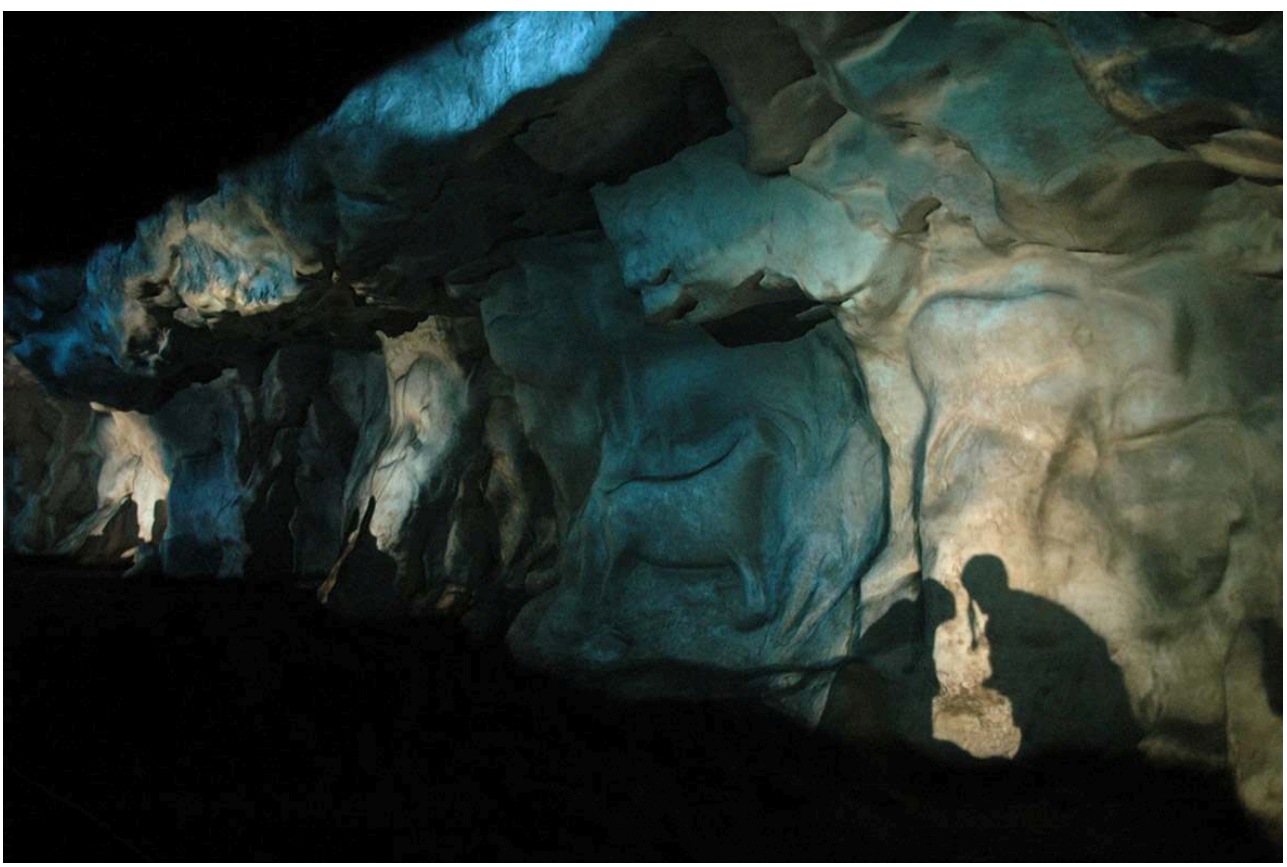

Centre d'interprétation du Roc-aux-Sorciers à Angles-sur-l'Anglin (Vienne)

(c) Roc-aux-Sorciers

5 Quelques années après la reprise de ces recherches et leur publication à destination de la communauté scientifique ou au travers de conférences pour le grand public, la Communauté de Communes des Vals de Gartempe et Creuse prend conscience de la valeur et surtout de l'originalité de ce patrimoine unique. Cependant, le site est fermé au public pour des raisons de conservation. Or, le souhait est de faire partager à un public aussi large que possible l'originalité et la puissance qui se dégagent de ce site magdalénien. C'est ainsi qu'est envisagée la création d'un Centre d'Interprétation organisé autour d'une restitution de la frise encore in situ (fig. $\mathbf{n}^{\circ} \mathbf{2}$ ).

6 La base de données 3D élaborée à des fins de recherche est alors exploitée dans le cadre de ce nouveau projet à destination du grand public. Cette variété d'exploitation fut possible car le cahier des charges pour l'obtention de cette base de données 3D a permis d'élaborer une ressource numérique adaptée aux usages qui en seront faits ${ }^{9}$.

\section{Les exploitations de la base de données 3D}

\section{L'usage scientifique à l'origine de la base de données 3D}

7 L'appréhension de la troisième dimension est devenue l'une des exigences essentielles du relevé d'art pariétal paléolithique. Cette question tombe sous le sens pour les œuvres sculptées mais aussi pour les peintures et les gravures qui adoptent les reliefs de la paroi sur lesquels elles s'appuient. Une figure pariétale est ainsi rarement plane, que son volume soit donné par sa technique d'exécution, ou suggéré par la surface accidentée de la paroi. Ainsi, les travaux menés ces dernières années témoignent d'une nouvelle approche de la 3D comme technique d'acquisition et d'archivage des données 
particulièrement efficace, et comme instrument d'analyse et support de diffusion innovant et pertinent.

8 Après la photogrammétrie, les cartes morphologiques en courbe de niveaux et la méthode des profils lumineux, l'enregistrement laser tridimensionnel est la dernière technique développée dans la recherche attachée à l'étude de l'art pariétal pour la retranscription de la troisième dimension. Sa première application dans ce domaine se fait en 1994 à la grotte Cosquer (Portmiou, Bouches-du-Rhône). Le premier critère d'usage de cette ressource est la restitution (le fac-similé) et non directement la réponse à des problématiques de la recherche.

Le relevé topographique et l'enregistrement 3D des œuvres pariétales - sculptées et gravées - du Roc-aux-Sorciers ont été réalisés, depuis 1999, dans le cadre des recherches autorisées par le Ministère de la Culture et de la Communication ${ }^{11}$. En effet, la capture par le laser est particulièrement appropriée pour la restitution des reliefs, positifs pour la sculpture ou négatifs pour la gravure. Au Roc-aux-Sorciers, le recours au laser 3D est adapté à l'enregistrement des figures sculptées et gravées et aux problématiques de recherches que soulèvent ces œuvres. Ce site témoigne donc d'une utilisation tout à fait originale de l'enregistrement laser tridimensionnel dans l'étude de l'art pariétal paléolithique, notamment dans l'étude de l'évolution de la paroi et des œuvres qui y sont sculptées et gravées, dans la restitution des parties détruites ou manquantes ou encore d'homothéties particulières aidant à reconstituer les différentes étapes de la frise ${ }^{12}$.

10 Le besoin de disposer d'une base de données 3D a donc été soulevé en tout premier lieu par les scientifiques, dès la prise en charge de la reprise de l'étude du Roc-auxSorciers ${ }^{13}{ }^{14}$.

\section{L'enregistrement laser tridimensionnel au Roc-aux- Sorciers}




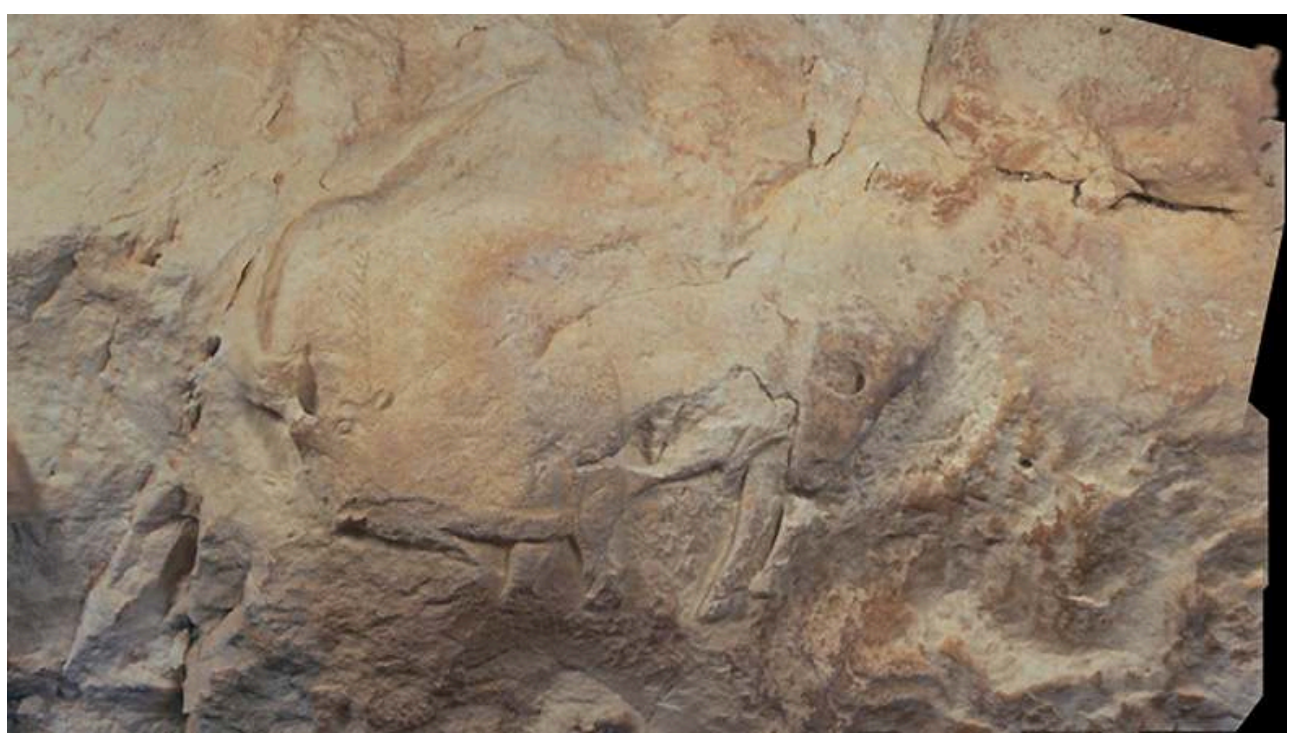

Relevé laser 3D du bison de la Cave Taillebourg, Le Roc-aux-Sorciers à Angles-sur-l'Anglin (Vienne) (C) Geneviève Pinçon, Ministère de la Culture et de la Communication, réalisation Art graphique et patrimoine

11 Les premiers essais d'enregistrement laser 3D des œuvres pariétales sur place au Rocaux-Sorciers sont réalisés en 1999 sur la paroi de la Cave Taillebourg où un bison sculpté est encore en place au plafond ${ }^{15}$ (fig. $\mathbf{n}^{\circ} 3$ ). Cet enregistrement a été effectué avec une précision de 5 dixièmes de millimètres ${ }^{16}$. Entre 2001 et 2003 a lieu une deuxième campagne de relevé in situ qui permet une saisie particulièrement fine de l'ensemble des zones gravées de l'abri Bourdois. La saisie est faite avec une précision de 1 à 2 dixièmes de millimètres pour les zones gravées. Parallèlement, la saisie des zones sculptées s'est poursuivie en adoptant une précision de 5 à 8 dixièmes de millimètres. L'année 2004 voit l'élargissement du champ d'application du laser 3D avec les premiers enregistrements de blocs ornés effondrés de la Cave Taillebourg, à partir desquels sont élaborés des prototypes en résine et réalisés les premiers raccords numériques des blocs $^{17}$. En 2005 et 2006, la saisie tridimensionnelle de la globalité de l'abri Bourdois (du sol à la paroi surplombant l'abri) avec une précision de 2 à 5 millimètres, vient compléter l'environnement des zones ornées (fig. $\mathbf{n}^{\circ} \mathbf{4}$ ). Elle s'accompagne d'un géoréférencement topographique. 
Figure 4

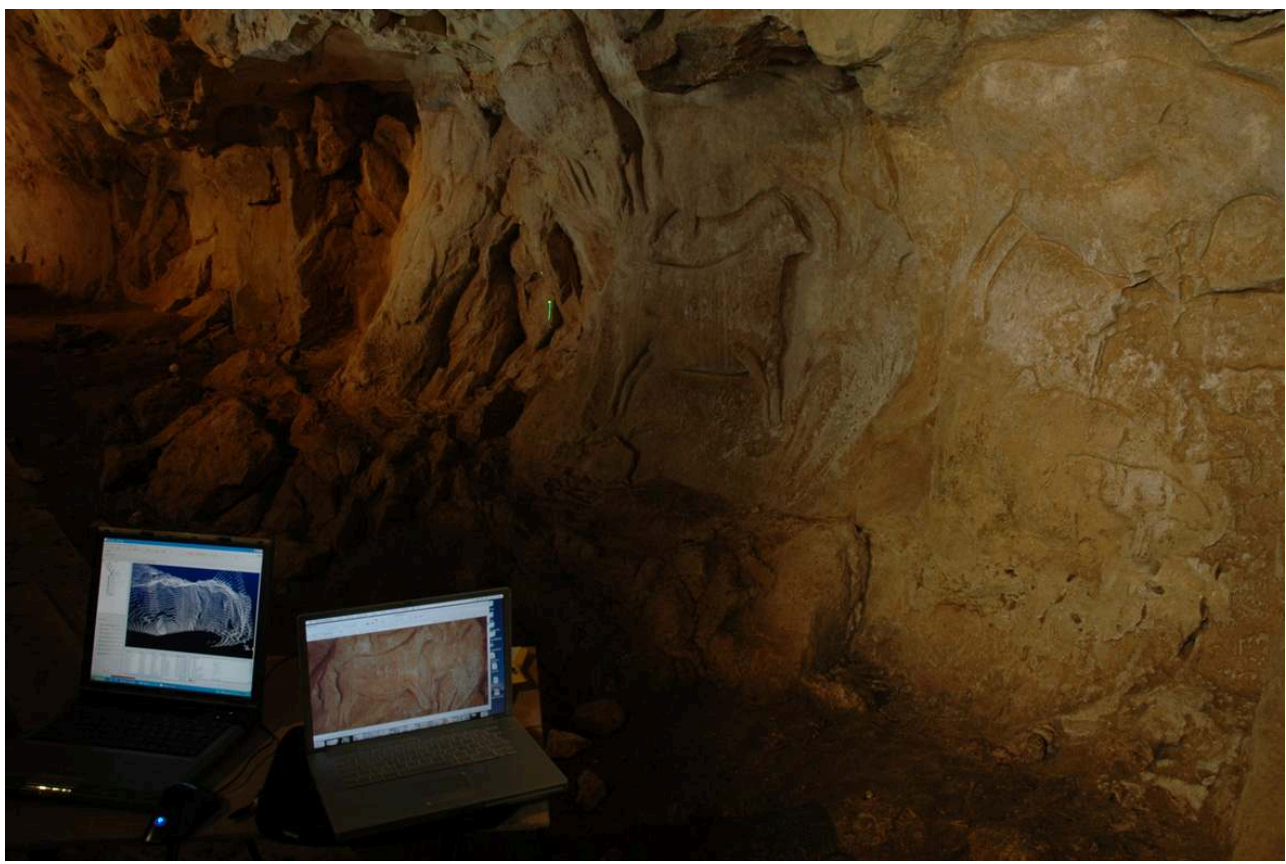

Relevé au laser de la paroi de l'abri Bourdois, Le Roc-aux-Sorciers à Angles-sur-l'Anglin (Vienne) Phot. Philippe Plailly. (C) Geneviève Pinçon, Ministère de la Culture et de la Communication, réalisation Art graphique et patrimoine

12 Le modèle numérique de l'ensemble de l'abri - de précision variable, selon l'intérêt archéologique des secteurs - est ainsi disponible depuis la fin de l'année $2006^{18} 1920$.

\section{Les éléments du cahier des charges pour la base de données 3D du Roc-aux-Sorciers}

Objectifs 
Figure 5

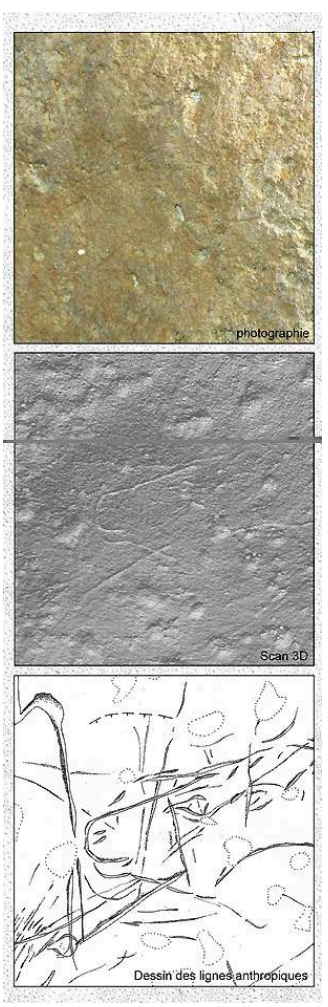

Relevé au laser des gravures pariétales de l'abri Bourdois, Le Roc-aux-Sorciers à Angles-sur-l'Anglin (Vienne)

(C) Geneviève Pinçon, Ministère de la Culture et de la Communication, Art graphique et patrimoine

Le relevé laser tridimensionnel vise à acquérir et reconstruire des surfaces, qu'elles soient naturelles ou anthropiques. Cet enregistrement est réalisé selon un protocole répondant à des objectifs et à des besoins précis. Au Roc-aux-Sorciers, le but est d'obtenir un enregistrement pertinent, exhaustif et fidèle de la sculpture et de la gravure pariétale.

Le relevé laser tridimensionnel est parfaitement adapté aux œuvres sculptées et permet d'obtenir une transcription de la troisième dimension sans distorsion dans les dimensions, dans les proportions, ni dans la position relative réelle des œuvres. Son échelle de résolution étendue lui permet de saisir avec une grande précision les différentes nuances du tracé depuis la gravure fine jusqu'au haut-relief. Un rendu fidèle des figures, des formes générales jusqu'aux détails les plus ténus est très utile dans notre analyse archéologique, notamment pour les zones gravées (fig. $\mathbf{n}^{\circ} \mathbf{5}$ ). En tant que procédé technique systématique, il produit des documents bruts sans hiérarchisation des informations et permet la construction d'un référentiel objectif, outil indispensable pour le chercheur puis pour la restitution de son analyse. Le cahier des charges fut donc dans le cas du Roc-aux-Sorciers proposé et rédigé par les archéologues. 


\section{Niveau de précision}

15 Le niveau de précision déterminé dans le cahier des charges fixe le niveau d'analyse et le degré de précision de l'information à saisir. Au Roc-aux-Sorciers, trois niveaux de précision ont été définis selon la problématique archéologique :

- zone naturelle : 2 à 5 millimètres ;

- zone anthropique sculptée : 5 à $8 / 10$ de millimètres ;

- zone anthropique gravée : 1 à $2 / 10$ de millimètres.

Ces degrés de précision établis selon les zones sous-entendent une connaissance du terrain ou l'accompagnement $\mathrm{du}$ scientifique mais aussi une formalisation des problématiques de la recherche pour lesquelles la base de données 3D est incontournable.

17 Si l'enregistrement laser tridimensionnel est particulièrement bien adapté aux œuvres sculptées, la conjonction de plusieurs méthodes d'enregistrement - sélectionnées en fonction des caractéristiques matérielles de l'œuvre, de l'environnement et de la précision recherchée - reste indispensable pour obtenir un résultat satisfaisant.

\section{Mode d'acquisition et type de fichiers attendus ${ }^{21}$}

Le cahier des charges précisait la mise à disposition des fichiers bruts sous forme de nuages de points et de fichiers de points de coordonnées $\mathrm{x}$, y et $\mathrm{z}$, restitués dans des formats les plus ouverts possible (formats ASCI, OBJ et DXF). Était également sollicité le découpage de ce fichier source en plusieurs fichiers de moindre taille afin d'en faciliter une manipulation de façon segmentaire. Le modèle numérique de surface était également attendu sous la forme d'un fichier type 3D susceptible d'être utilisé dans différentes applications. Pour la base de données complète et globale du site, les différents nuages de points devaient être géoréférencés. Parallèlement, des clichés photographiques numériques de qualité devaient venir compléter les films de la caméra numérique ${ }^{22}$.

\section{Reconstitutions 3D attendues}

19 Un Modèle Numérique de Surface (MNS) était également attendu résultant de l'assemblage des fichiers de scans partiels sur un logiciel de modeling 3D, puis le remesh (adaptation du nombre de points à la géométrie de la surface numérisée), le maillage, au plus près des points, réalisé selon le degré angulaire des surfaces générées, et le mapping (habillage en textures de la base), conçu avec les clichés numériques de qualité.

La précision de corrélation entre la grille du modèle 3D et les pixels de l'image était fondamentale pour une lecture réelle du modèle avec sa texture en images réelles. Selon l'usage (patrimonial, scientifique), différentes qualités d'images devaient pouvoir être utilisées. 
Figure 6

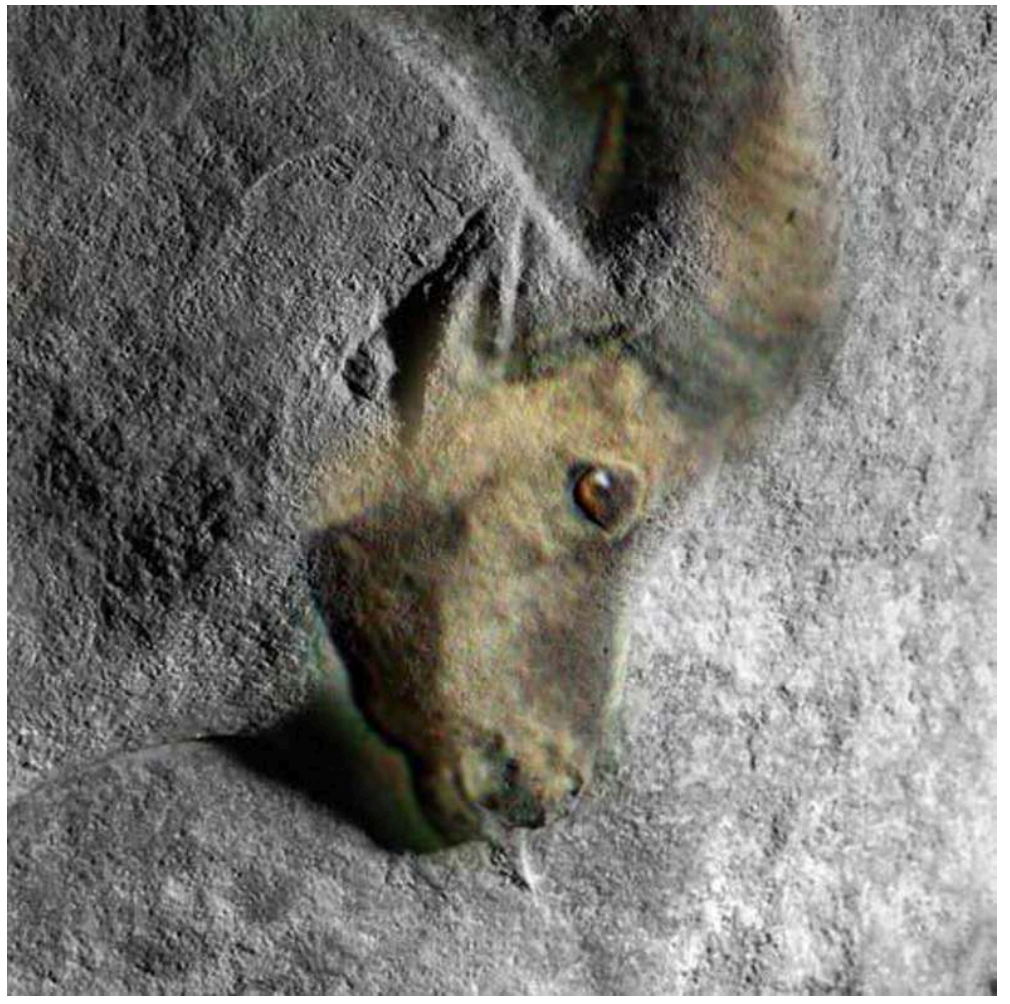

Métamorphose de la tête de bouquetin mâle sculptée en tête de bouquetin vivant, simulation (c) Olivier Auber

21 Enfin, ayant également d'emblée le désir de transmettre nos observations sur la paroi de façon dynamique (notamment la question des retailles des sculptures), la base de données devait permettre de restituer des reconstitutions hypothétiques pour expliciter l'évolution de la paroi au cours du Magdalénien ou permettre des anamorphoses (fig. $\left.\mathbf{n}^{\circ} \mathbf{6}\right)$. La réalisation de plusieurs petites vidéos thématiques a ainsi été prévue dans le cahier des charges.

Le recours au laser 3D n'a pas été systématique et obligatoire. L'investissement dans ce procédé est étudié au cas par cas selon les objectifs à atteindre et la nature de la restitution attendue. Le choix de l'outil s'est fait par rapport aux objectifs et aux moyens conjugués dont nous disposions. C'est grâce à un échange constant avec le prestataire que le résultat obtenu put être adapté à ce rapport fondamental: hauteur de l'investissement technique (coût, niveau technique à déployer) / impact du résultat (ponctuel et à peu de portée scientifique ou au contraire résultat d'intérêt général pour l'étude et d'un grand apport pour l'analyse et la compréhension de l'œuvre).

\section{Applications scientifiques des modèles numériques tridimensionnels}

Plusieurs problématiques scientifiques, depuis la composition du décor pariétal et son insertion dans la topographie générale de l'abri, jusqu'à l'étude stylistique et technique des œuvres, peuvent être abordées grâce à différents traitements sur la base de données $3 \mathrm{D}^{23}$. Le modèle numérique 3D offre la possibilité de travailler à différentes 
échelles, sur tout ou partie de la base de données. Cette segmentation dépend des choix opérés par le chercheur, et donc de son exploitation personnelle du modèle numérique par rapport à sa problématique ${ }^{24}$. posé une réflexion collective sur la manière de restituer un tel patrimoine. Pour les élus et les scientifiques, la frise devait être au cœur du projet. L'accès à l'original 
n'étant plus possible, il fallait permettre à tous de vivre l'expérience émouvante et personnelle de la découverte de cet art sculpté monumental.

Pour les élus il s'agissait de restituer la frise sculptée magdalénienne dans son état connu. C'est dans ce sens qu'ils rencontrèrent l'équipe scientifique, avant toute initiative de projet, la sollicitant pour disposer d'un état de la connaissance et des éléments fondateurs d'un projet pour le public.

Mais, outre la restitution de la frise magdalénienne au grand public, la volonté des élus était, à travers ce projet, de contribuer au développement local des Vals de Gartempe et Creuse en enrichissant l'offre culturelle et économique, et d'augmenter les retombées financières des prestataires locaux sur ce territoire.

C'est dans ce sens qu'une étude économique du territoire et de ses potentiels touristiques fut sollicitée par la communauté de communes en partenariat avec les services de l'état afin de pouvoir disposer d'une estimation financière de l'investissement possible pour un tel projet sans engager les acteurs publics dans des dépenses d'investissement et de fonctionnement excédant leurs moyens. C'est ainsi que l'enveloppe globale budgétée pour l'ensemble de ce projet fut évaluée à hauteur de 2,5 3 millions d'euros ${ }^{26}$.

Figure 7

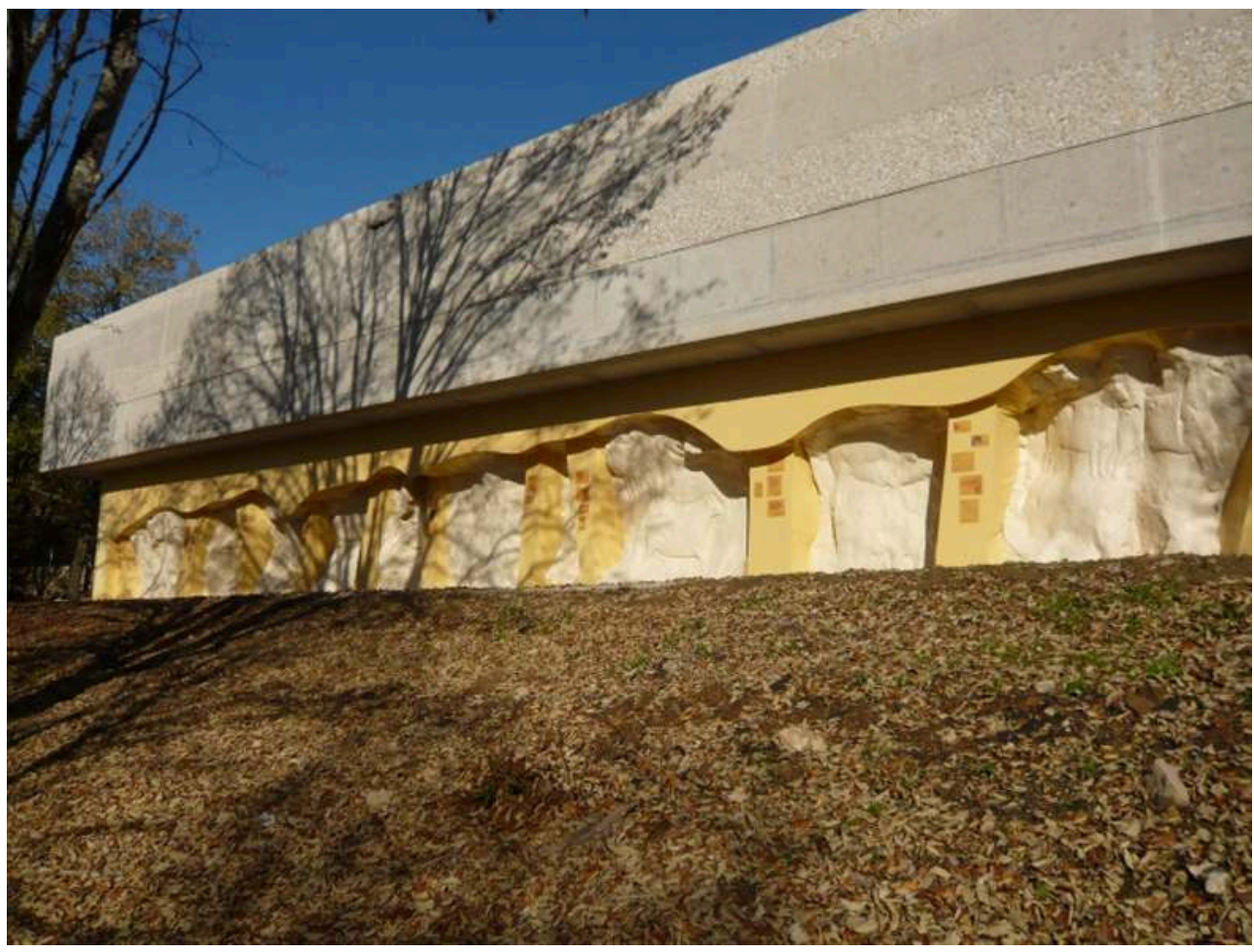

Présentation de la frise sculptée en extérieur

(c) Roc-aux-Sorciers

La Communauté de Communes des Vals de Gartempe et Creuse (maître d'ouvrage), entourée d'un comité de pilotage et d'un comité scientifique ${ }^{27}$, a élaboré son cahier des charges en précisant sa volonté d'offrir au grand public une image de l'époque magdalénienne qui lui soit accessible. L'utilisation du numérique était bien sûr un des éléments de la modernité d'un tel projet et était mentionnée dans l'appel d'offre. 
Figure 8

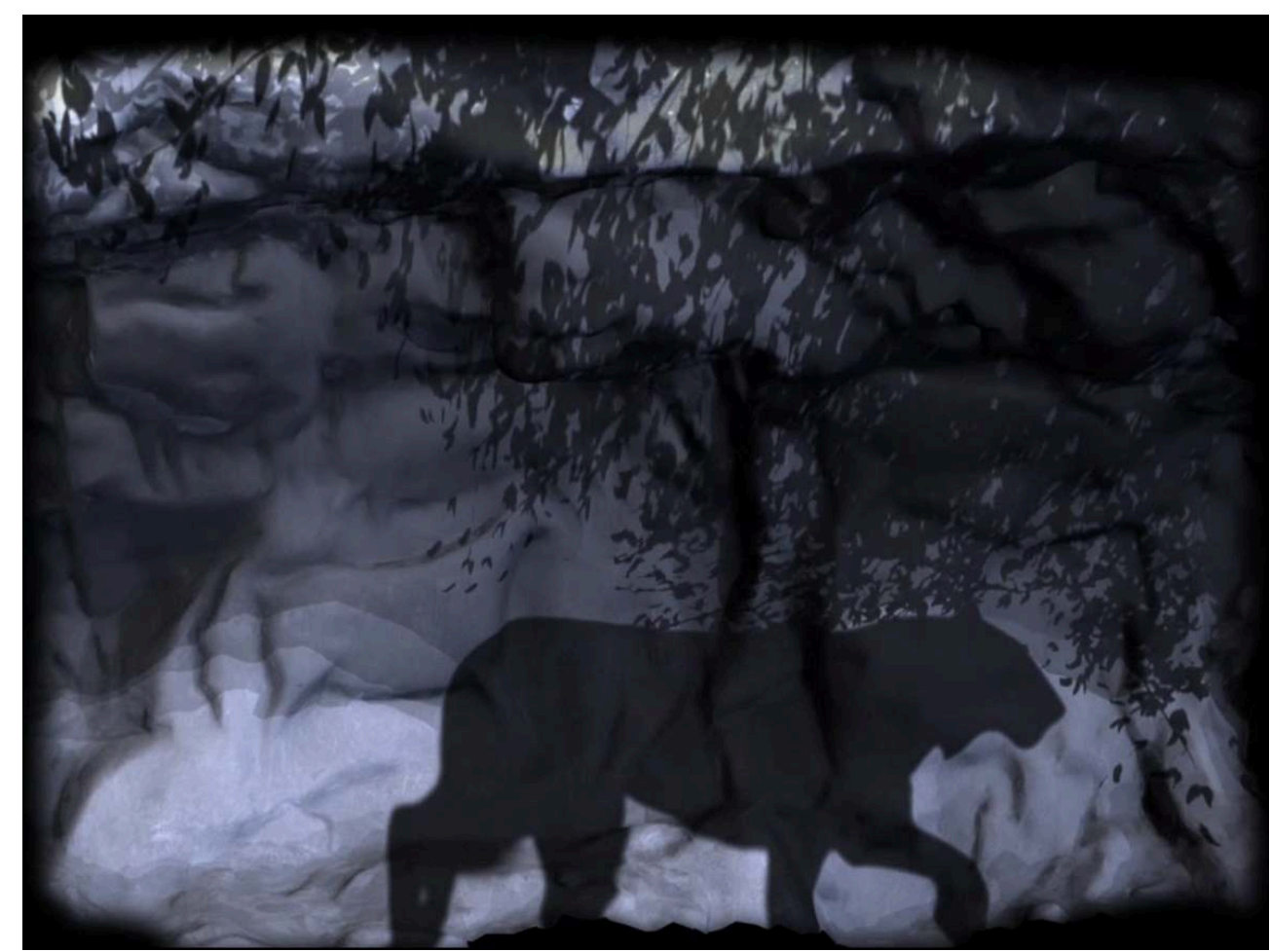

Image extraite de la scénographie

(c) Roc-aux-Sorciers

Le choix de la maîtrise d'œuvre s'est porté sur l'équipe Paulet-Auber-Comte-Hoge pour une proposition dont l'originalité était de proposer deux formes complémentaires de restitution de la frise sculptée, privilégiant l'objet même du projet, à savoir la frise magdalénienne, dans une architecture discrète et un environnement naturel, adaptés au lieu protégé (site classé au titre des monuments historiques et des sites) où le projet allait être implanté. D'une part une forme " archéologique » (fig. $\mathbf{n}^{\circ} 7$ ) révélant la frise dans ses moindres détails, que le visiteur peut toucher et apprécier dans un contexte naturel face à la vallée. D'autre part une forme « onirique » - support de projection s'adressant au visiteur sur le registre sensible de l'imaginaire ${ }^{28}$ (fig. $\mathbf{n}^{\circ} \mathbf{8}$ ).

\section{La frise numérique exploitant la 3D : parti pris et éléments de conception de la maîtrise d'œuvre}


Figure 9

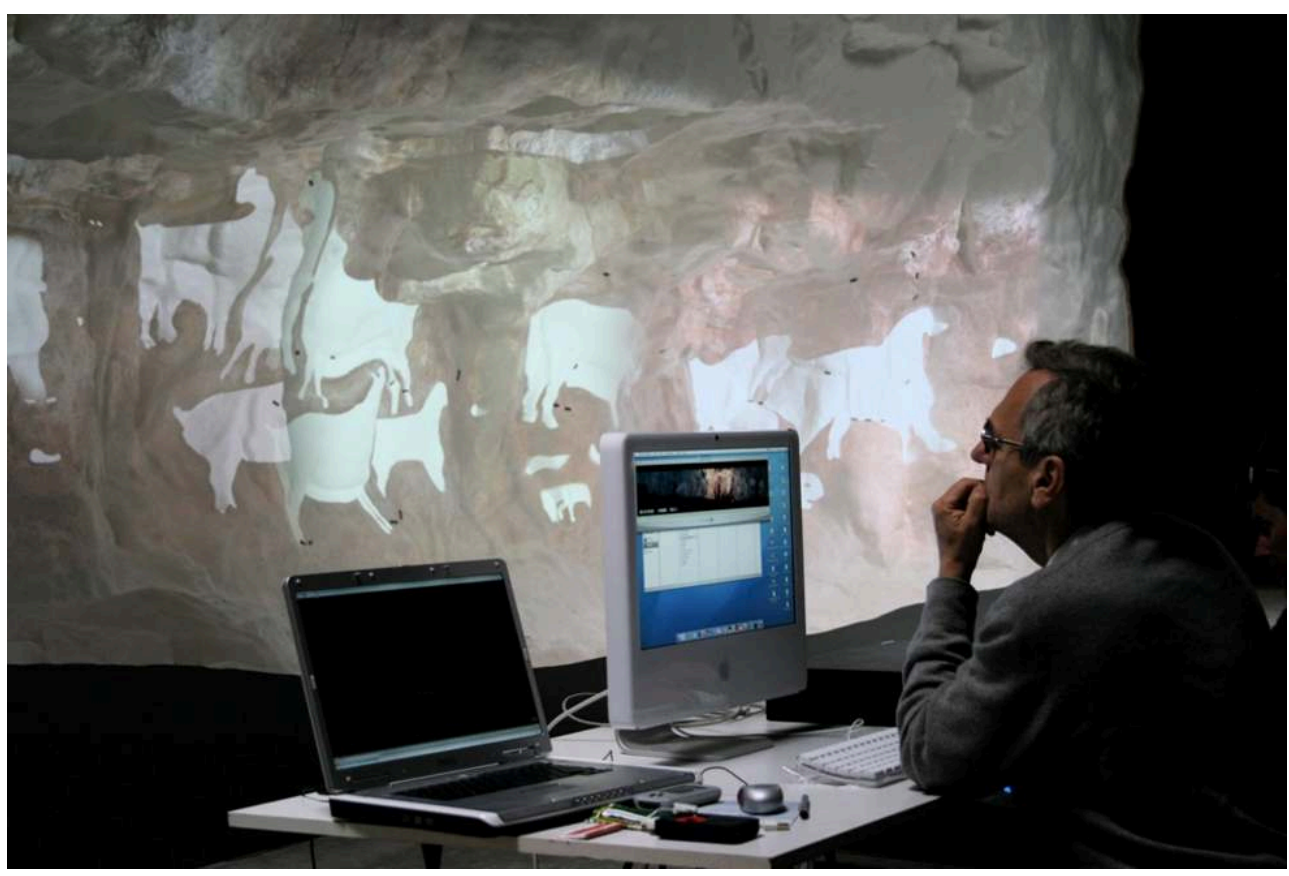

Mise en scène sonore par Patrick Marchand

(C) Roc-aux-Sorciers

La frise restituée grandeur nature à partir de la base numérique 3D est mise en scène dans un spectacle d'une trentaine de minutes proposant une découverte scientifique et onirique de l'art pariétal sculpté. Les visiteurs plongés dans le noir vivent une expérience unique, un voyage dans l'onirique, le scientifique, l'imaginaire et deviennent les propres acteurs de la découverte. La frise est révélée par des jeux de mise en scène, mêlant son et image, le tout spatialisé (fig. $\mathbf{n}^{\circ} \mathbf{9}$ ).

C'est la mise en commun de compétences multiples qui a permis cette réalisation.

La société Art Graphique et Patrimoine a effectué un relevé laser en 3D de la frise originale pour la recherche qui a été utilisé pour la fabrication du fac-similé en polystyrène par commande numérique (atelier HA).

La technique de prototypage par usinage à été choisie pour la reproduction à l'échelle $1 / 1$ de la paroi car elle a permis de réaliser directement la frise dans la matière définitive (Polystyrène M1) à partir de la numérisation 3D d'origine. Cette technique

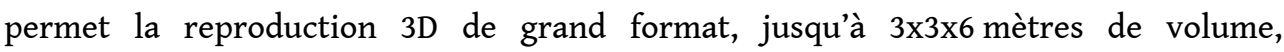
parfaitement compatible au fort relief du Roc-aux-Sorciers. Pour permettre un usinage et un assemblage cohérents et stables des blocs composant l'ensemble, un calcul de calepin d'appareil a été réalisé avec une optimisation préalable du fichier 3D original pour adapter la maille de l'usinage final. Le résultat ne devait pas être un fac-similé classique mais un écran de projection 3D extrêmement fidèle géométriquement à l'original, sans qu'il soit besoin de reproduire tous les détails puisqu'ils sont restitués par les images projetées. On s'est donc appuyé sur un maillage optimisé d'environ $5 \mathrm{~mm}$, pour les zones anthropiques, jusqu'à $2 \mathrm{~cm}$, pour les zones moins sensibles (falaise), sachant que lorsqu'on optimise, il y a plus de points de maillage dès que le relief est accidenté. Le maillage doit être fermé, à facettes non croisées avec la même orientation et sans superposition pour obtenir un fichier «.stl » prêt pour l'usinage 
(fig. $\mathbf{n}^{\circ}$ 10). À partir du scan, un grand travail de post-production est nécessaire pour pouvoir décliner la même base pour différents usages.

Figure 10

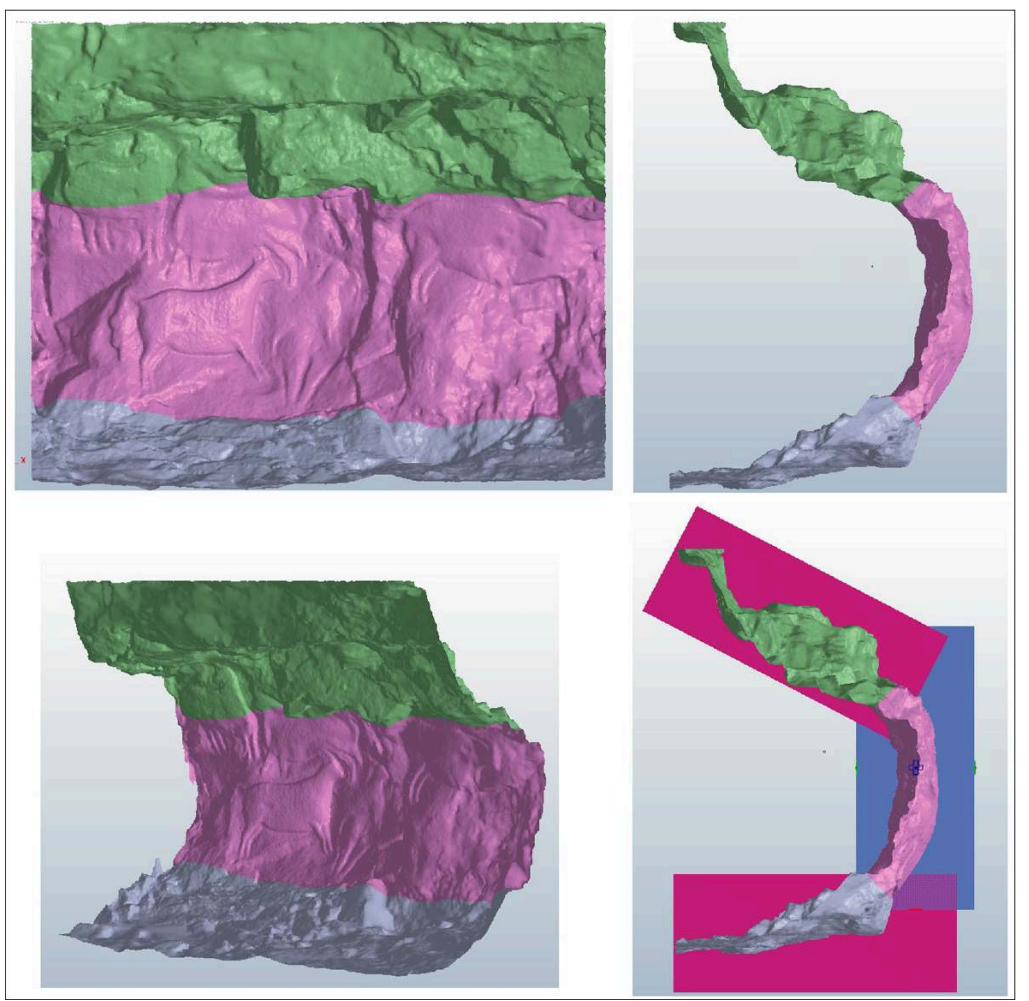

Préparation du fichier pour l'usinage

(c) Geneviève Pinçon, Ministère de la Culture et de la Communication, Art graphique et patrimoine

Dans ce cas l'emploi du modèle 3D pour l'ensemble de la réalisation est très important car la paroi posée, assemblée après usinage et la projection des images de synthèse, doivent être parfaitement raccordées, d'où l'utilité du même modèle 3D référent. L'usinage pour la reproduction s'appuie sur les coordonnées $\mathrm{x}, \mathrm{y}, \mathrm{z}$ des fichiers fournis, sans déformation, interprétation ou interpolation (nonobstant les dilations physiques possibles et mesurables). Les calculs d'images de synthèse respectent également les mêmes coordonnées.

41 Nous avons donc l'utilisation de la même base de données 3D à des fins scientifiques, techniques et de mise en valeur, d'où l'importance d'une bonne rédaction du cahier des charges en vue de permettre ces optimisations de mise en œuvre et de coûts.

L'État a autorisé l'utilisation de l'image du site, des archives, des clichés et de la base de données 3D pour réaliser ce projet.

La société AXYS est ensuite intervenue pour habiller en 3D anamorphosée (600 000 images calculées) ce support en relief et permettre la mise en place de la scénographie. Pour éviter la pollution sonore, la société IEC, qui a assuré l'ingénierie audiovisuelle du site, a réparti dans l'espace les systèmes de diffusion gérés par un Médialon V4 (12 haut-parleurs MBP 50 Amadeus, 4 ensembles de basses et satellites et 4 caissons de basse). 
diversité, auquel le Centre d'Interprétation apporte un enrichissement certain. Ainsi, i ne fait pas de doute que le Centre d'Interprétation pose les premiers jalons d'une nouvelle manière d'aborder la valorisation des sites ornés et du patrimoine. Ce nouveau Centre d'Interprétation propose de rendre accessible un patrimoine en exploitant les résultats les plus récents de la recherche ainsi que les technologies les plus novatrices à travers l'exploitation de la base de données 3D au service des publics.

\section{Conclusion}

$\mathrm{Au}$ Roc-aux-Sorciers, la base de données 3D réalisée dans un contexte scientifique trouve un usage d'ordre archivistique mais également touristique. En effet la connaissance de l'objet à numériser et les questions qu'il pose à la recherche orientent la constitution et la forme de cette ressource. Elles mettent en exergue les zones «sensibles» (à connaître et faire connaître) de l'objet étudié et permettent ainsi également des restitutions au public. Il ne s'agit pas de solliciter de façon systématique

In Situ, 13 | 2010 
une base de données 3D exhaustive et de grande précision sur l'objet à étudier. Plusieurs autres modes de restitution peuvent répondre à un grand nombre de besoins d'archivage ou d'étude et de restitution, comme la photographie, le dessin ou le film. Mais quand les problématiques de la recherche conduisent, comme c'est le cas au Rocaux-Sorciers, à l'utilisation de la 3D, il apparaît logique que ce même outil puisse aider à la restitution au grand public et puisse être partagé avec la communauté scientifique ou avec les restaurateurs chargés de la conservation de ce patrimoine.

\section{Encart}

\section{Les implications du choix d'un mode de restitution à partir d'une base de données 3D : l'exemple de la frise sculptée de la Chaire-à-Calvin en Charente $^{30}$}

Figure 11

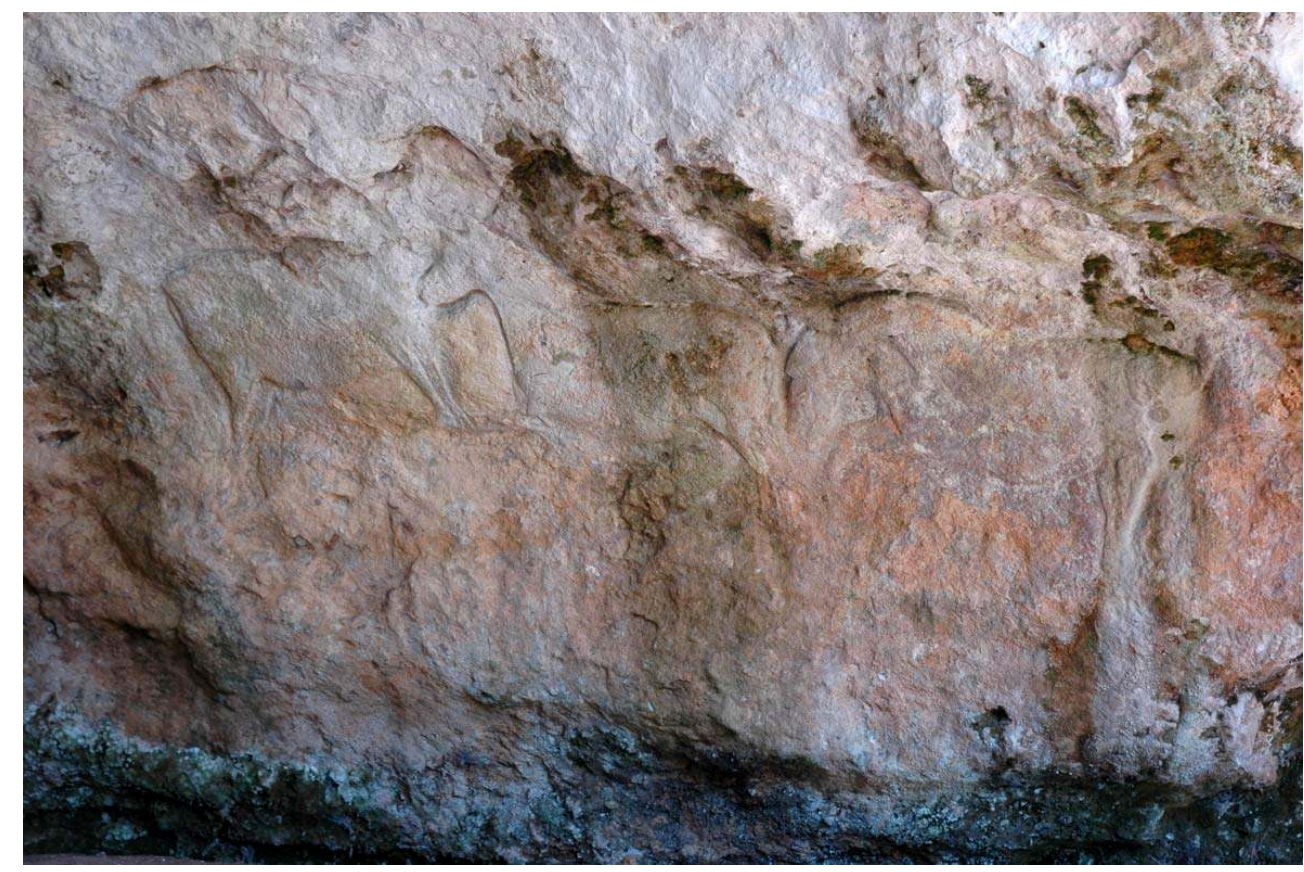

Frise sculptée de la Chaire-à-Calvin, Mouthiers-en-Boëme (Charente)

(c) Geneviève Pinçon, Conseil général de Charente, phot. Alain Maulny

En 2006, parallèlement aux travaux menés au Roc-aux-Sorciers, à la demande du Conseil général de Charente et avec son soutien financier, nous avons appliqué à l'étude de l'art pariétal de la Chaire-à-Calvin, à Mouthiers-sur-Boëme en Charente (fig. $\left.\mathbf{n}^{\circ} \mathbf{1 1}\right)$ la même méthodologie d'enregistrement 3D que celle mise en œuvre et développée à l'abri du Roc-aux-Sorciers, à savoir :

- un relevé global géoréférencé de l'abri et des parois aux abords, avec une précision de 2 à 5 millimètres

- une saisie de précision de la petite zone sculptée actuellement connue, avec une précision de 5 à 8 dixièmes de millimètres. 
Figure 12

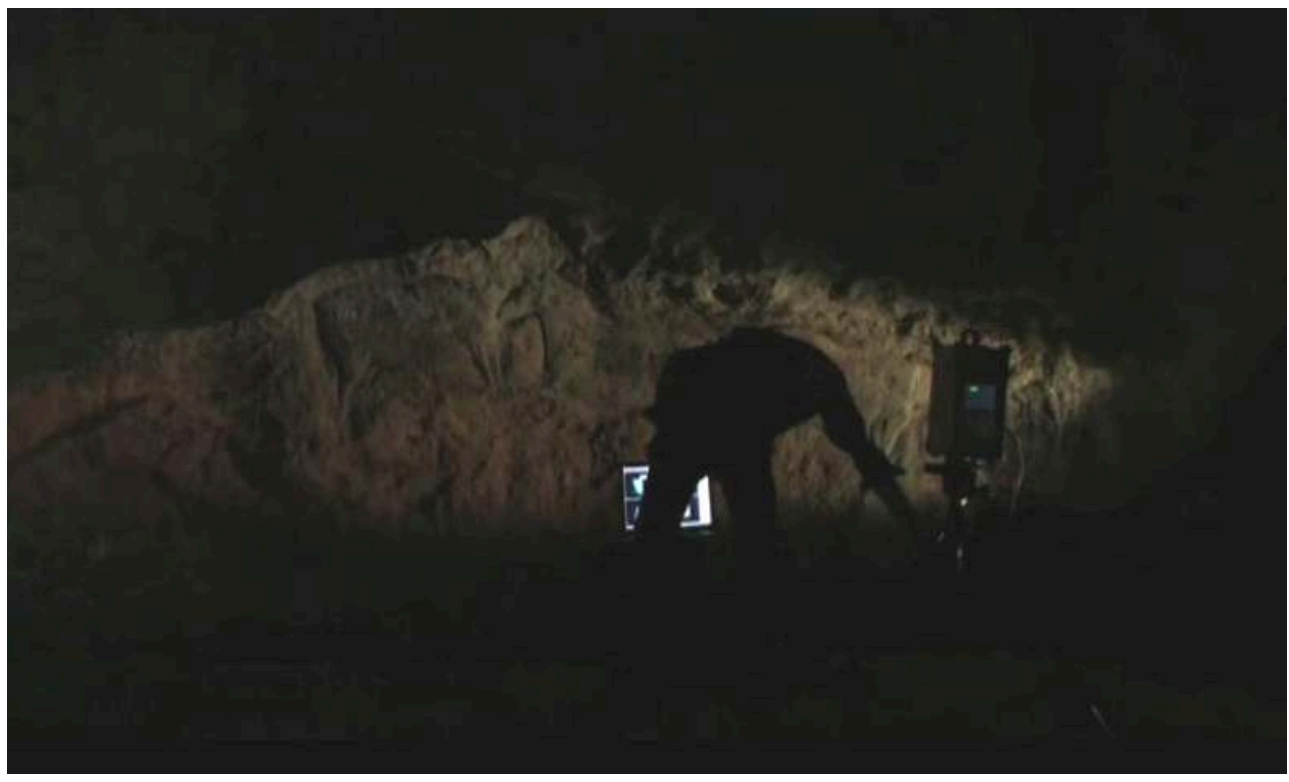

Numérisation de l'abri sculpté de la Chaire-à-Calvin

(c) Conseil général de la Charente, Art graphique et Patrimoine, Philippe Plailly, Geneviève Pinçon

51 Le modèle numérique de l'ensemble de l'abri a été livré au Conseil général de la Charente, maître d'ouvrage du projet de numérisation, début 2007, accompagné d'applications de visualisation du modèle 3D que nous avons définies (fig. $\mathbf{n}^{\circ} \mathbf{1 2}$ ).

En 2009, à l'initiative du Conseil général de la Charente, une restitution en pierre de la frise à une échelle réduite fut entreprise sur le site en vue d'une présentation au public, notamment malvoyant, dans le cadre de la valorisation du site de la Chaire-à-Calvin. Pour réaliser cette restitution une nouvelle base de données 3D fut réalisée sur la proposition du prestataire alors retenu pour ce projet.

53 Sensible à cette démarche, il nous semble intéressant de faire état de ces réalisations exploitant différemment les bases de données 3D.

\section{De la connaissance à la médiation : quelle restitution envisager ?}


Figure 13

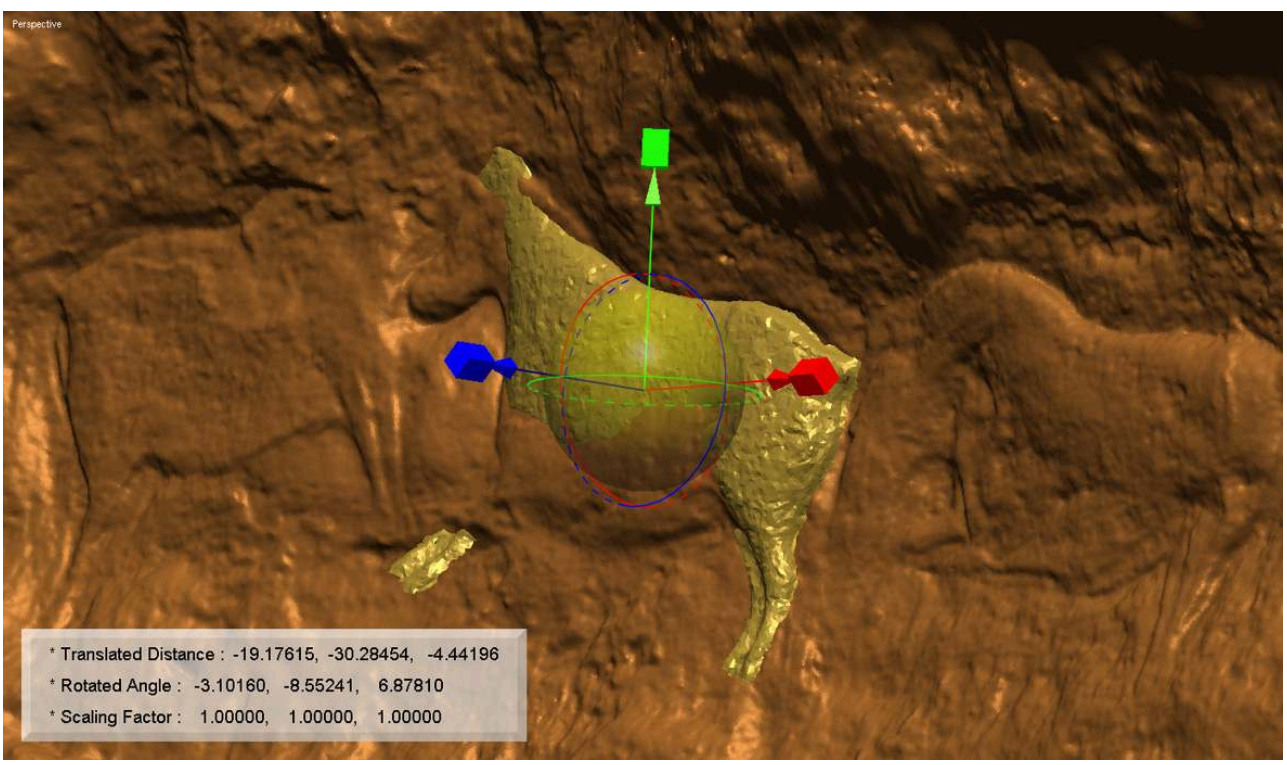

Comparaison homothétique de deux formes animalières, I'une du Roc-aux-Sorciers, l'autre de la Chaire-à-Calvin

(C) Geneviève Pinçon, Ministère de la Culture et de la Communication, Conseil général de la Charente, Art graphique et Patrimoine

Lors de l'étude de l'art pariétal sculpté magdalénien de la Chaire-à-Calvin, l'analyse de la paroi montra un état de conservation très différent de la paroi et donc des œuvres magdaléniennes selon les secteurs. Ces observations conduisirent à de nouvelles interprétations des figures à partir des zones les mieux conservées ${ }^{31}{ }^{32}$. L'exploitation des bases de données 3D réalisées sur la frise de la Chaire-à-Calvin et sur celle du Rocaux-Sorciers a permis de conforter ces nouvelles hypothèses d'interprétation (fig. $\left.n^{\circ} 13\right)$.

55 Au moment de l'étude pour la valorisation du site, un prototypage automatique à une échelle choisie, sans interprétation car issu de la lecture du fichier, fut proposé. Ce projet aurait mis en exergue les résultats de la recherche à la portée du public. Le public, y compris malvoyant, en touchant une matière reproduisant avec exactitude la frise sculptée, aurait pu ainsi saisir les différents états de conservation de la paroi et suivre le chercheur dans son cheminement d'analyse et de ré-interprétation des œuvres. 
Figure 14
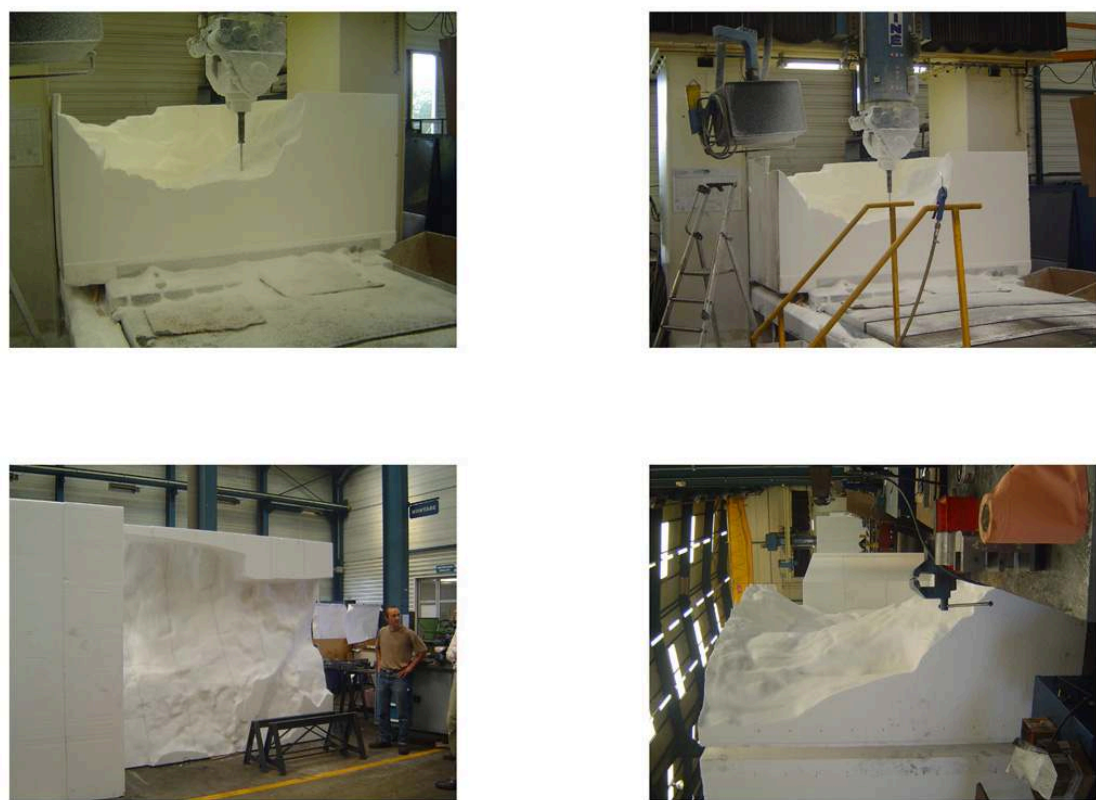

Usinage de la restitution exacte à l'échelle 1 depuis la base de données 3D

(c) Roc-aux-Sorciers

L'utilisation de la base de données 3D réalisée dans le cadre de la recherche aurait pu être faite, comme dans le cas du Roc-aux-Sorciers, pour obtenir une reproduction fidèle à la précision de la numérisation (fig. $\mathbf{n}^{\circ} \mathbf{1 4}$ ). La reproduction devant être à une échelle réduite, la numérisation initiale se trouvait être suffisamment précise et compatible avec les techniques de prototypage direct pour fournir une copie fidèle, hormis la réduction d'échelle. Cependant, la numérisation initiale de 2 à 5 dixièmes de millimètres ne semblant pas compatible avec la technique de reproduction du prestataire retenu par le maître d'ouvrage pour le projet de valorisation, une nouvelle numérisation à été réalisée pour reproduire le panneau orné. 
Figure 15

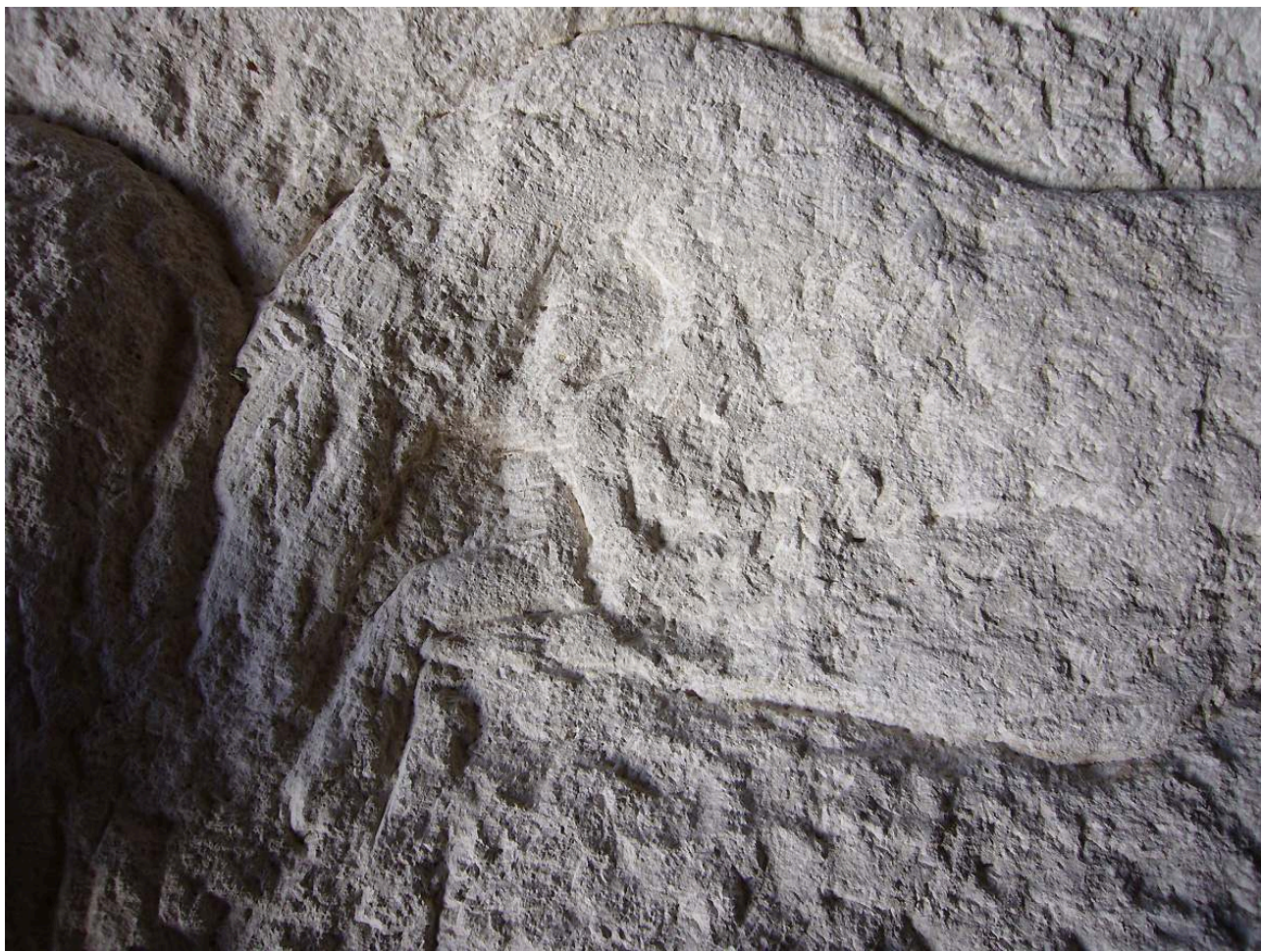

La Chaire-à-Calvin, création à l'échelle 2/3 avec un bras relié à la base de données 3D (geste du sculpteur assisté)

Phot. Geneviève Pinçon. (c) Geneviève Pinçon

Pour effectuer une copie fidèle à partir de fichiers très denses et précis, seules des techniques de prototypage direct de grande précision doivent être envisagées (stéréolithographie, usinage...). Or le processus retenu dans le projet de valorisation de la Chaire-à-Calvin implique la main de l'homme dans la chaîne, ce qui altère et rend inutile la précision du scanner 3D. La main humaine ne peut pas restituer avec la même précision (relative ou globale) que la machine assistée par ordinateur et retire à la copie ainsi réalisée son intérêt scientifique. Le choix retenu pour la valorisation du site fut de restituer la frise en calcaire local à une échelle réduite où certes la matière est fidèle par rapport à l'original mais pas les altérités de l'épiderme de la paroi originale. Un sculpteur maniant un bras relié à la base de données, créa une interprétation de l'objet. Cette sculpture porte tous les stigmates de cette intervention manuelle. Les traces de fraisage sur toute la surface ne rendent pas l'aspect exact de la paroi, et limitent donc l'explication et le partage des interprétations des œuvres magdaléniennes proposées par les scientifiques (fig. $\mathbf{n}^{\circ} \mathbf{1 5}$ ). Une telle restitution non conforme à l'original est intéressante pour offrir des interprétations mais elle constitue une copie d'œuvre non conforme à l'original. C'est pourquoi elle ne semble pas adaptée à une restitution d'ordre scientifique, recherchée dans ce domaine.

Cette expérience nous alerte sur la nécessité de travailler en équipe et d'accompagner les projets de valorisation de tout patrimoine ayant fait l'objet d'une recherche. 


\section{in Institut national du patrimoine}

Communication présentée lors du séminaire ART RUPESTRE : LA 3D UN OUTIL DE MÉDIATION DU RÉEL INvisıBLE ? qui s'est tenu du 4 au 6 juin 2008 à Angles-sur-l'Anglin, coordonné par l'Institut national du patrimoine, avec la participation de la direction de l'architecture et du patrimoine

\section{NOTES}

1. Traduction du résumé par Paul Bahn, que nous remercions.

2. Rousseau, Lucien. Le Magdalénien dans la Vienne. Découverte et fouille d'un gisement du Magdalénien à Angles-sur-l'Anglin (Vienne). Bull. de la Société Préhistorique Française, t. 30, 1933, p. 239-256.

3. Saint-Mathurin, Suzanne de. Les bas-reliefs et la frise sculptée d'Angles-sur-l'Anglin (Vienne). Institut Français d'Anthropologie, séance du 15 mars 1950, p. 6-8.

4. Saint-Mathurin, Suzanne de, GARROD, Dorothy. L'abri du Roc-aux-Sorciers (Angles-surl'Anglin, Vienne). Congrès Préhistorique de France, $n^{\circ}$ 15, Poitiers-Angoulême, 1956, p. 89-94.

5. Saint-Mathurin, Suzanne de. L'abri du Roc-aux-Sorciers, Angles-sur-l'Anglin, Vienne. L'art des cavernes. Atlas des grottes ornées paléolithiques françaises, Paris: Imprimerie Nationale, 1984, p. 583-587.

6. Iakovleva, L., Pinçon, Geneviève. Angles-sur-l'Anglin. La frise sculptée du Roc-aux-Sorciers. Paris : éd. R.M.N. et C.T.H.S., 1997.

7. Pinçon, Geneviève (dir.). Le Roc-aux-Sorciers : art et parure du Magdalénien. Paris : RMN, 2009, disponible sur le site : http://www.catalogue-roc-aux-sorciers.fr.

8. Voir dans ce numéro : Pinçon, Geneviève, Bourdier, Camille, Fuentes, Oscar, Abgrall, Aurélie. De la manipulation des images 3D. [Document électronique]. Paris : Ministère de la Culture et de la Communication.

9. Pinçon, Geneviève. Méthodes de relevé appliquées à l'étude de l'art pariétal sculpté magdalénien du Roc-aux-Sorciers (Angles-sur-l'Anglin, France). Bulletin AAPC, n 33-2004, p. 41-58.

10. Bourdier, C., Fuentes, O., Hamon, G., Pinçon, G. « Technologies 3D appliquées à la sculpture pariétale magdalénienne", Images et relevés archéologiques, de la preuve à la démonstration, sous la direction d'O. Bouchsenchutz, coll Actes des Congrès des sociétés historiques et scientifiques, $132^{\mathrm{e}}$ Congrès national des sociétés savantes historiques et scientifiques, Arles, 2007, CDRom.

11. Avec le soutien financier de l'État, de la Région Poitou-Charentes et du Conseil Général de la Vienne que nous remercions vivement.

12. Bourdier, C., Fuentes, O., Hamon, G., Pinçon, G. « Technologies 3D appliquées à la sculpture pariétale magdalénienne", Images et relevés archéologiques, de la preuve à la démonstration, sous la direction d'O. Bouchsenchutz, coll Actes des Congrès des sociétés historiques et scientifiques, $132^{\mathrm{e}}$ Congrès national des sociétés savantes historiques et scientifiques, Arles, 2007, CDRom. 
13. Auzanne, I., Desroches, E., Pinçon, G. «Bilan d'interventions sur le site magdalénien du Rocaux-Sorciers à Angles-sur-l'Anglin (86, France) : restauration, analyse de la polychromie et relevé numérique 3D ", In : L'Art avant l'Histoire, la conservation de l'art préhistorique, $10^{\mathrm{e}}$ journée d'études de la Section Française de l'Institut International de Conservation (SFIIC), Paris, 23-24 mai 2002, p. 221-241.

14. PINÇON, Geneviève. Chronologie des œuvres magdaléniennes du Roc-aux-Sorciers (Anglessur-l'Anglin): entre tradition et innovation. In Situ, revue des patrimoines [document électronique], 2008, $\mathrm{n}^{\circ}$ 9. Paris : Ministère de la Culture et de la Communication.

15. Auzanne, I., Desroches, E., Pinçon, G. «Bilan d'interventions sur le site magdalénien du Rocaux-Sorciers à Angles-sur-l'Anglin (86, France) : restauration, analyse de la polychromie et relevé numérique 3D ", In : L'Art avant l'Histoire, la conservation de l'art préhistorique, $10^{\mathrm{e}}$ journée d'études de la Section Française de l'Institut International de Conservation (SFIIC), Paris, 23-24 mai 2002, p. 221-241.

16. Pinçon, Geneviève. Méthodes de relevé appliquées à l'étude de l'art pariétal sculpté magdalénien du Roc-aux-Sorciers (Angles-sur-l'Anglin, France). Bulletin AAPC, n 33-2004, p. 41-58.

17. Ibid.

18. Ibid.

19. Ibid.

20. Pinçon, G., Fuentes, O., Bourdier, C. « Les sculptures pariétales magdaléniennes du Roc-auxSorciers (Vienne) et de la Chaire-à-Calvin (Charente) : œuvres d'un groupe culturel ou d'un seul et même artiste?", In: Virtual Restrospect 2007, Actes du colloque de Pessac (Gironde), 14-16 novembre 2007, Bordeaux : Ausonius, coll. Archéovision, volume 3, p. 13-20.

21. Pour la présentation des éléments techniques 3D, se reporter à l'article: Bourdier, C., Fuentes, O., Hamon, G., Pinçon, G. «Technologies 3D appliquées à la sculpture pariétale magdalénienne ", Images et relevés archéologiques, de la preuve à la démonstration, sous la direction d'O. Bouchsenchutz, coll Actes des Congrès des sociétés historiques et scientifiques, $132^{\mathrm{e}}$ Congrès national des sociétés savantes historiques et scientifiques, Arles, 2007, CDRom.

22. Réalisés par Alain Maulny, photographe, ces clichés sont numérisés et enregistrés selon les normes préconisées par le Ministère de la Culture. Voir le site : (http://www.culture.gouv.fr/ culture/mrt/numerisation/fr/f_04.htm). Ibid.

23. Abgrall, A., Bourdier, C., Fuentes, O., Pinçon, G. «Le relevé graphique analytique : un outil indispensable ", In : Buisson-Catil, J., Primault, J. (dir.). Préhistoire entre Charente et Vienne. Hommes et sociétés du Paléolithique. Chauvigny: Association des Publications Chauvignoises, mémoire XXXVIII.

24. Voir dans ce numéro : Pinçon, Geneviève, Bourdier, Camille, Fuentes, Oscar, Abgrall, Aurélie. De la manipulation des images 3D. [Document électronique]. Paris : Ministère de la Culture et de la Communication.

25. Ibid.

26. Le financement du projet, d'un montant réel de 2,8 millions d'euros, a été possible grâce aux subventions de l'État, de la Région Poitou-Charentes, du Département de la Vienne, de la Communauté de Communes des Vals de Gartempe et Creuse, de la commune d'Angles-surl'Anglin, mais aussi des crédits Européens. Enfin, il faut aussi souligner le mécénat de la fondation du Crédit Agricole.

27. Les membres du comité scientifique: Geneviève Pinçon, Oscar Fuentes, Camille Bourdier, Jacqueline Lorentz, Jacques Jaubert, Jean-Pierre Gély, Catherine Schwab, Sergio Ripoll, Jacques Tarrête, Gerhard Bosinski, Jose Antonio Lasheras, Sophie Tymula, Paul Bahn, Jean Clottes, JeanMichel Geneste, François Levêque, Sandrine Costamagno, Véronique Dujardin, Jacques BuissonCatil, Marie-Pierre Rigomier-Chollet. 
28. Fuentes, O., Pinçon, G. Le Roc-aux-Sorciers: Du site au centre d'interprétation, un choix scénographique original. Les Nouvelles de l'archéologie, n 117, octobre 2009.

29. Les travaux du centre d'interprétation du Roc-aux-Sorciers ont débuté en septembre 2006 et se sont achevés fin 2007. Le site ouvrit officiellement ses portes au public, par délégation de service public, le vendredi 21 mars 2008.

30. Geneviève Pinçon, Directrice scientifique des recherches conduites sur l'art pariétal à la Chaire-à-Calvin, UMR 7041 Arscan, genevieve.pincon@culture.gouv.fr

31. Pinçon, G. et coll. La sculpture en abri : un art partagé ? Les Dossiers d'Archéologie, $n^{\circ} 324$, novembre-décembre 2007, p. 86-91.

32. Pinçon, G., Bourdier, C. Nouvelle interprétation d'une sculpture pariétale de la Chaire-àCalvin (Charente, France) : apport de la technologie 3D. International Newsletter on Rock Art, Inora, $\mathrm{n}^{\circ}$ 54-2009, p. 11-16.

\section{RÉSUMÉS}

La base de données 3D réalisée dans le cadre de la recherche archéologique sur les sculptures pariétales magdaléniennes (il y a 15000 ans) a pu être exploitée également pour une reproduction grandeur nature des œuvres à destination du grand public dans le Centre d'interprétation du Roc-aux-Sorciers à Angles-sur-l'Anglin (Vienne, France). Cette réalisation originale est présentée par les différents acteurs du projet (maître d'ouvrage, maître d'œuvre, scientifiques, prestataire 3D) offrant ainsi le déroulé de la mise en œuvre de la restitution à partir de la base de données 3D et faisant part des éléments du cahier des charges pour l'enregistrement laser tridimensionnel dont l'exploitation s'est avérée répondre à des utilités variées. Quand les problématiques de la recherche conduisent à utiliser la 3D, il paraît logique que ce même outil puisse aider à la restitution au grand public et puisse être partagé avec la communauté scientifique ou avec les restaurateurs chargés de la conservation de ce patrimoine, comme le montre cette réalisation. Les implications du choix d'un mode de restitution à partir d'une base de données 3D sont également évoquées avec l'exemple de la frise sculptée magdalénienne de la Chaire-à-Calvin (Charente, France) dans le cadre d'un projet de valorisation.

The creation of a 3D database within the framework of archaeological research on Magdalenian parietal sculptures $(15,000$ years old) has also been usable for a full-size reproduction of the figures for the benefit of the general public in the Interpretation Centre of Le Roc-aux-Sorciers at Angles-sur-l'Anglin (Vienne, France). This unique production is presented by the various participants in the project (contracting authority, prime contractor, scientists, 3D provider), thus providing the whole story of the reconstruction's implementation from the 3D database, and providing information on the specifications for 3-dimensional laser recording, the use of which proved to be useful in a variety of ways. When research problems lead one to find solutions through utilisation of 3D. it appears logical that this same tool might help in reconstructions for the general public, and may be shared with the scientific community or with restorers in charge of the conservation of this heritage; that is what this production demonstrates. The implications of the choice of a reconstruction method from a 3D database are also evoked through the example of the Magdalenian sculpted frieze of La Chaire-à-Calvin (Charente, France) within the framework of a valorisation project. 


\section{INDEX}

Keywords : 3D database, parietal sculptures, Magdalenian, general public, Interpretation Centre, Roc-aux-Sorciers, Angles-sur-l'Anglin, 3D, reconstruction, specifications, 3-dimensional laser recording, restoration of heritage, conservation of heritage, Chaire-à-Calvin, valorisation, 3D reconstruction, 3D scenography, tourism

Mots-clés : base de données 3D, sculptures pariétales, Magdalénien, grand public, Centre d'interprétation, Roc-aux-Sorciers, Angles-sur-l'Anglin, 3D, restitution, cahier des charges, enregistrement laser tridimensionnel, restauration du patrimoine, conservation du patrimoine, Chaire-à-Calvin, valorisation, reconstitution 3D, scénographie 3D, tourisme

\section{AUTEURS}

\section{GENEVIÈVE PINÇON}

Directrice scientifique des recherches conduites sur le gisement du Roc-aux-Sorciers, UMR 7041 Arscan.genevieve.pincon@culture.gouv.fr

\section{OSCAR FUENTES}

Directeur du centre d'interprétation du Roc-aux-Sorciers, UMR 7041 Arscan. oscar.fuentes@rocaux-sorciers.com

\section{RENÉ BARRÉ}

Maire de la Roche-Posay, délégué à la communauté de communes des Vals de Gartempe et Creuse, ancien Président de la communauté de communes des Vals de Gartempe et Creuse, porteur du projet du Roc-aux-Sorciers.rpm.barre@wanadoo.fr

\section{OLIVIER AUBER}

Maître d'œuvre pour la partie multimédia du centre d'interprétation du Roc-aux-Sorciers. olivier.auber@km2.net

\section{GAËL HAMON}

Directeur d'exploitation - Société Art Graphique et Patrimoine, réalisation de la base de données 3D.g.hamon@artgp.fr 